\title{
Quecksilberverschluß statt Kork oder Kautschuk bei der organischen Analyse;
}

\author{
von

\section{J. Marek.}

Die zum Verbinden des Wasserabsorptionsapparates mit dem Verbrennungsrohre gebräuchlichen Kork- oder Kautschukstopfen (Kautschukschlauch) sind bekanntlich mit einigen Mängeln behaftet, welche teils chemischer, teils physikalischer Natur sind. Hierunter hat oft bei sonst korrekter Ausführung der Verbrennung die besonders bei unbekannten und hochmolekularen Verbindungen so sehr erwünschte Genauigkeit der Wasserstoff- und Kohlenstoffbestimmung zu leiden. Ob sich die von Ditmar vorgeschlagenen, eigens dazu hergestellten Kautschukstopfen ${ }^{1}$ ) besser bewähren werden, ist abzuwarten.

Meiner Ansicht nach ist es am besten, wenn man für den obigen Zweck die Verwendung von Kork und Kautschuk ganz ausschlieBt. Als Ersatz dafür empfiehlt sich ein QuecksilberverschluB. Es würde aber sehr schwer fallen, aus dem schwer schmelzbaren Glase der Verbrennungsröhre einen angeschmolzenen QuecksilberverschluBteil herzustellen. Mun fertigt sich daher einen solchen aus dem gewöhnlichen Glase und befestigt ihn dann mittels einer solchen Abdichtung, welche luftdicht schlieBt, einer Hitze von etwa $300^{\circ}$ vollkommen widersteht und sehr dauerhaft ist. Ein solches Abdichtungsmittel ist Asbest, welcher mit geschmolzenem Silberchlorid überzogen wird.

$\mathrm{Zu}$ diesem Zwecke zieht man das Verbrennungsrohr zu einer etwa $4-5 \mathrm{~cm}$ langen, nicht zu engen offenen Spitze aus, umwickelt das Ende derselben mit so viel Asbestwolle oder Asbestpapier, dab sie in der mit dem Quecksilberverschlußteil versehenen und mit Tressensilber ${ }^{2}$ ) fast vollgefüllten Knieröhre $a$, Fig. 1, festsitzt. Sodann wird die abgedichtete Stelle

1) Gummi-Ztg. 20, 465; durch Chem. Centralbl. 1906, I, 1287.

2) Erhältlich bei Franz $\mathrm{Hugershoff-Leipzig.}$ 
Marek: Quecksilberverschlub statt Kork etc. 181

der Röhre an einer nicht zu kleinen Flamme vorsichtig erhitzt und die Verbrennungsröhre so nach oben gerichtet, daB man auf den Asbest nach und nach die nötige Menge von Silberchlorid bringen kann. Dieses schmilzt man mittels des Lötrohres so zusammen, dab die ganze äußere Fläche der Asbestdichtung mit geschmolzenem Silberchlorid bedeckt ist. Zuletzt erhitzt man dieses noch mit der leuchtenden Flamme des Brenners bis zum gleichmäBigen Schmelzfluß und läßt erkalten. Sollte die gekittete Stelle noch nicht luftdicht sein, so wird die Röhre schwach evakuiert und gleichzeitig das Silberchlorid bis zum Schmelzen erhitzt, wodurch dasselbe tiefer in den Asbest eindringt. In dieser Weise wird eine tadellose luftdichte Abdichtung erzielt. Man stopft nun die Spitze des Verbrennungsrohres mit Tressensilber voll, und zwar bis etwas über die Stelle, wo die Verjüngung derselben beginnt.

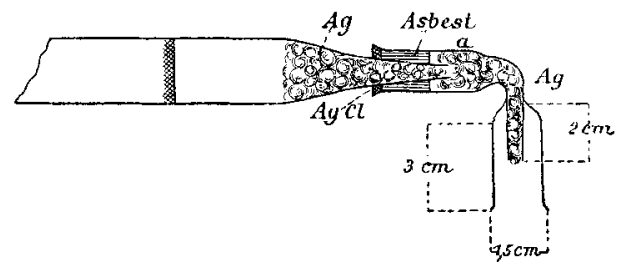

Fig. 1.

Das Tressensilber hat den Zweck, während der Analyse die Wärme gut weiter zu leiten, um auf diese Weise das sich sonst dort ansammelnde Wasser zu verdampfen. Will man aber bei wasserstoffreichen Substanzen sicher gehen, da $B$ alles Wasser aus der Spritze der Verbrennungsröhre und der angesetzten Knieröhre entfernt ist, so kann man unter diese eine Asbestdrahtnetzrinne legen und darunter eine kleine Flamme stellen. Die Erwärmung wird die Abdichtung nicht schädlich beeinflussen.

Statt sich das erwähnte Knierohr anzufertigen, kann man einfacher die Spitze des Verbrennungsrohres auf bekannte Weise rechtwinkelig biegen und an diese das für den QuecksilberverschluB nötige Röhrchen $b$ (Fig. 2) mittels Asbests und Silberchlorids befestigen. In diesem Falle muß man aber den 
182 Marek: QnecksilberverschlnB statt Kork etc.

Asbestpfropfen an den beiden freiliegenden Seiten mit Silberchlorid überziehen.

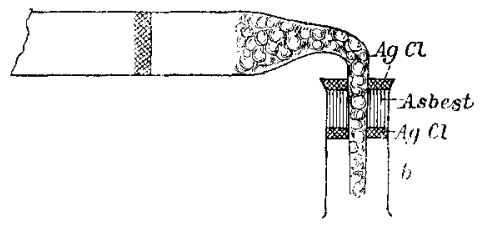

Fig. 2.

Um das auf die eine oder andere Art hergestellte Ver. brennungsrohr mittels Quecksilbers mit dem Wasserabsorptionsapparate verbinden zu können, muB man auch das Ansatzrohr dieses Apparates in der aus der Fig. 3 ersichtlichen Weise etwas abändern.

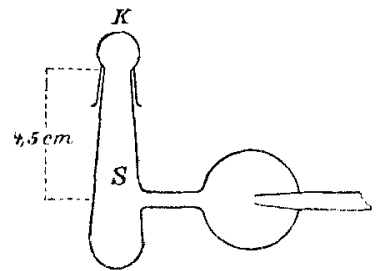

Fig. 3.

Hier ist $\boldsymbol{K}$ eine kleine aufgeschliffene Glaskappe, welche bei der Wägung des Absorptionsapparates verwendet wird.

Soll die Verbindung zwischen dem Verbrennungsrohre und dem Wasserabsorptionsapparate hergestellt werden, so befestigt man auf das konische Röhrchen $S$ (Fig. 3 u. 4) mittels eines Korkes (welcher - wie leicht ersichtlich - die Genauigkeit der Resultate nicht beeinflussen kann) ein etwa $2,5 \mathrm{~cm}$ breites und etwa $5 \mathrm{~cm}$ langes Glasrohr $R$ (Fig. 4), führt nachher die Spitze des Verbrennungsrohres so weit als

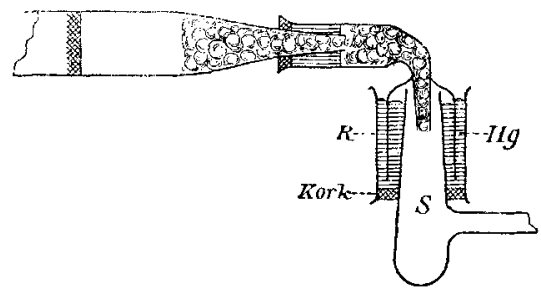

Fig. 4 . 
Marek: Quecksilberverschluß statt Kork etc. 183 möglich in das Röhrchen $S$ ein und gieBt in den Mantel $R$ die nötige Menge trockenen und reinen Quecksilbers.

Man könnte zwar das Glasrohr $R$ an das Röhrchen $S$ anschmelzen; dies ist aber nicht zu empfehlen, da sich in diesem Falle - abgesehen davon, daB man dadurch das Gewicht des Apparates erhöhen würde - der Apparat von dem anhaftenden Quecksilber ziemlich umständlich reinigen lassen würde.

Den Wasserabsorptionsapparat muß man auf eine zweckmäBige Art stützen, damit er durch das Gewicht des Quecksilbers nicht schief zu hängen kommt, anderseits muB man aber entsprechend dafür sorgen, da $B$ sich das Verbrennungsrohr (als Folge der Wirkung des Auftriebes im Quecksilber) nicht event. nach oben biegen kann.

Nach beendeter Verbrennung wird zuerst der Kohlensäureabsorptionsapparat und dann der Wasserabsorptionsapparat entfernt, indem man den Verbrennungsrohransatz aus dem Mantel $R$ (Fig. 4) hebt. Das Quecksilber entfernt man, indem man den Apparat entsprechend neigt, oder indem man den Kork lüftet und das Quecksilber ausfließen läbt.

Bei der Verwendung von Bleisuperoxyd zur Absorption der Halogene, Schwefel- und Stickstoffoxyde benutzt man (wenn man es nicht vorzieht, das Verbrennungsrohr unmittelbar mit dem Bleisuperoxyde zu beschicken) eine entsprechend weitere und längere Knieröhre, welche so mit Bleisuperoxyd beschickt wird, $\mathrm{da} B$ dieses von beiden Seiten mit Tressensilber begrenzt ist. Diese Röhre wird dann auf die erwähnte Weise mit dem Verbrennungsrohre verbunden (Fig. 5).

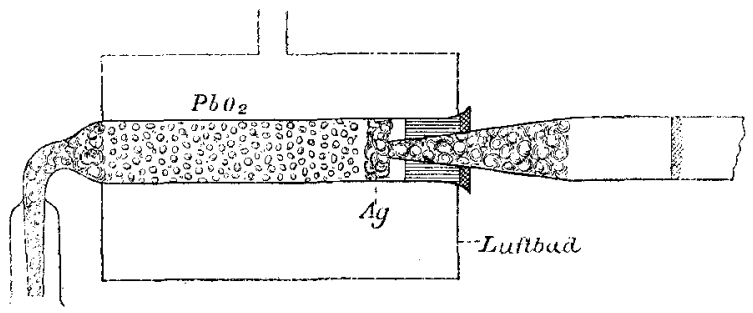

Fig. 5.

MuB man aus irgend einem Grunde die Verbrennungsröhre von der Knieröhre trennen, so braucht man die abge- 
184 Marek: QuecksilberverschluB statt Kork etc.

dichtete Stelle nur genügend zu erhitzen, bis das Silberchlorid geschmolzen und gröBtenteils abgetropft ist. Die Spitze des Verbrennungsrohres kann dann aus dem Knierohre herausgezogen werden.

Dieser QuecksilberverschluB ist ein relativ bequemes Verbindungsmittel, welches stets tadellos schlieBt und die $\mathrm{Zu}$ sammenstellung der Apparate bei weitem nicht so starr macht, wie die Kork- oder Kautschukstopfenverbindung, wenngleich sich jene erst bei solchen Verfahren recht praktisch und bequem erweist, welche erlauben, in einer und derselben Röbre eine ziemlich große Anzahl von Verbrennungen auszuführen.

Agram, Juni 1907. 